\title{
Uso de la metodología EPIK para determinar la vulnerabilidad del agua subterránea a la contaminación en Yucatán, México
}

DOI: $10.22403 /$ UQROOMX/TYP09/03

\author{
Emilio Rodrigo Bolio Barrios \\ Santos Armando Cabrera Sansores \\ Universidad Autónoma de Yucatán \\ Francisco Bautista \\ Centro de Investigaciones en Geografia Ambiental \\ Universidad Nacional Autonoma de México \\ Julia Guadalupe Pacheco Ávila \\ Universidad Autónoma de Yucatán
}

\section{RESUMEN}

La metodología EPIK, con un enfoque ambiental, fue desarrollada para acuíferos kársticos y tiene en cuenta variables como la alta permeabilidad en roca caliza, la cubierta protectora (profundidad del suelo), la infiltración y el paisaje kárstico (grado de desarrollo).

El acuífero de Yucatán es de alta permeabilidad, y para determinar su vulnerabilidad a la contaminación, mediante revisiones bibliográficas se identificaron los valores de los parámetros del método EPIK (epikarst, cubierta protectora y paisaje kárstico), adaptándolos a las particularidades del estado. Para los valores de la infiltración, también se realizaron pruebas de campo empleando el método del doble anillo.

Posteriormente, se evaluó la vulnerabilidad mediante un análisis multicriterio. La mayoría de los paisajes geomorfológicos evaluados se clasificaron como muy vulnerables, pero esta clasificación se presentó en diversos grados de acuerdo con los factores EPIK considerados en los diferentes paisajes.

Palabras Yucatán, acuífero, karst, paisajes geomorfológicos, tipos de CLAVE suelo 


\section{Introducción}

El estado de Yucatán es un área que posee rasgos físicos especiales, entre los que destacan: la ausencia de cuerpos de agua superficial, las planicies y las expresiones kársticas, que hacen del acceso, el uso y la protección del agua subterránea temas de gran importancia. El análisis de estos conceptos permitiría determinar la vulnerabilidad del agua subterránea en Yucatán, por lo que este trabajo inició con la búsqueda de información acerca de los métodos existentes, encontrando uno elaborado especialmente para áreas kársticas: el EPIK. También se hallaron trabajos previos en los que se emplearon métodos distintos a EPIK, que pudieran no reflejar las particularidades del estado.Algunas peculiaridades de Yucatán, que hacen de él un laboratorio natural, son los diferentes estados de formación del karst; la presencia de hundimientos y elevaciones; los tipos de suelo existentes, así como su profundidad y sus propiedades; las diversas expresiones kársticas; la presencia del agua subterránea y la profundidad a la que se encuentra; las zonas geohidrológicas y los paisajes geomorfológicos. De tal suerte, es necesario probar la aplicación de métodos específicos para las condiciones kársticas, y así identificar los modelos que hagan posible un mayor entendimiento del ambiente para proteger el acuífero kárstico en las planicies yucatecas. La información generada permitirá un mejor aprovechamiento del recurso hídrico, ya que se logrará reconocer las zonas potencialmente más vulnerables para aquellas actividades que representan un menor o mayor impacto al ambiente; se determinarán zonas prioritarias de conservación de aguas subterráneas; se identificarán los ecosistemas con mayores riesgos por la degradación, y se propondrán estrategias para la conservación y el aprovechamiento sustentable de los recursos naturales existentes.

\section{Antecedentes}

En la última década, tanto en Yucatán como en otras partes del mundo, los gobiernos han dado a los acuíferos kársticos un estatus de alta prioridad de conservación (Daly et al., 2002: 34I). La toma de decisiones para decretar zonas de conservación hídrica y para regular las actividades humanas requiere información científica que la fundamente. El acuífero de Yucatán tiene la particularidad de ser kárstico, de alta porosidad, y con grietas, cenotes y 
cavernas, que le confieren alta permeabilidad, lo que ocasiona que sea altamente vulnerable a la contaminación antrópica.

Las metodologías e índices desarrollados para determinar la vulnerabilidad tienen por lo general un enfoque hidrogeológico, y consideran principalmente parámetros como la profundidad de la zona saturada y no saturada, la litología, la conductividad hidráulica, la recarga neta, la infiltración y la pendiente del terreno. Entre los índices más comunes destacan DRASTIC, GOD, SINTACS y PI, que no son específicos para acuíferos kársticos (Auge, 2004:86; Goldscheider, 2005: 558). Otros, como el EPIK (Doerfliger, Jeannin y Zwahlen 1999: I65), con una orientación ambiental, fueron desarrollados para acuíferos kársticos y tienen en cuenta la alta permeabilidad en roca caliza, la cubierta protectora (espesor del suelo), la infiltración y el paisaje kárstico (grado de desarrollo).

Pérez y Pacheco (2004: 4I) aplicaron en Yucatán las metodologías AVI, GOD y DRASTIC para determinar la vulnerabilidad del acuífero. Los resultados mostraron que DRASTIC fue la más aproximada para determinar la vulnerabilidad del acuífero a la contaminación antrópica, sin embargo, los resultados no son concluyentes porque el modelo no considera el suelo.

El estado de Yucatán está situado al sureste de México, en el norte de la Península de Yucatán; sus formaciones geológicas corresponden a calizas del Terciario, en las planicies, mientras que en la costa se localizan planicies con sedimentos del Cuaternario (Bautista et al., 2003: 29 y 2005: 34; Ihl et al., 2007: 300). La zona de estudio comprende gran parte de la entidad, las grandes planicies estructurales con leptosoles, cambisoles y luvisoles, la línea de costa con arenosoles y solonchack, $y$ las dolinas agrupadas con luvisoles y cambisoles (INEGI, 2002: 20; Bautista et al., 2003: 30). Los subtipos climáticos son: tropical subhúmedo seco $A w 0$, tropical subhúmedo intermedio $A w_{1}$ y semiárido seco $\mathrm{BS}_{0}$ y semiárido menos seco $\mathrm{BS}_{1}$ (Orellana et al., 1999: 163). La vegetación es de matorral de duna costera, manglar, selva baja caducifolia, pastizales y selva mediana subcaducifolia (Flores y Espejel, 1994: 40).

Las geoformas por estudiar son: I) planicie costera, donde los suelos asociados son el solonchak y el regosol; 2) planicie kárstica subhorizontal baja, con el litosol y la rendzina como suelos predominantes; 3 ) dolinas agrupadas, las cuales pueden dividirse en cerradas, cuando no tienen agua, y abiertas, cuando si la contienen, como las ubicadas en la zona geohidrológica llamada semicírculo de cenotes, donde forman un conducto de alta permeabilidad que 
transporta el agua subterránea del sur del estado hacia la costa; 4) planicie kárstica ondulada, que se distingue por su variedad de formas kársticas, especialmente cenotes profundos; por otra parte, la ondulación del terreno se relaciona con la separación de dolinas en diversos estados de desarrollo y con una gran diversidad de fracturas; en esta geoforma, los suelos preponderantes son el litosol, la rendzina y el cambisol; y 5) planicie kárstica subhorizontal media, con características semejantes a la planicie kárstica subhorizontal baja, pero en la que, debido a las condiciones climáticas y a la elevada humedad, se ha desarrollado la selva mediana subperennifolia, este paisaje corresponde a la zona geohidrológica conocida como planicie interior; los suelos asociados son el litosol y la rendzina (figura I) (Bautista et al., 2005: 34).

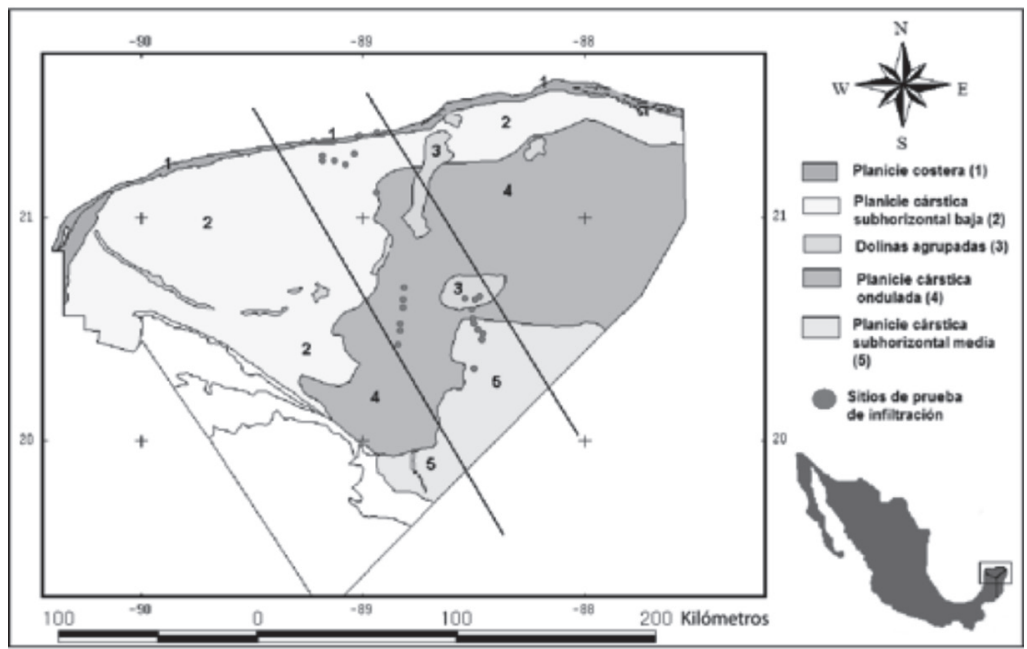

Figura I. Paisajes geOMORfológicos en LA zONA de ESTUdio

\section{Metodología}

Mediante una revisión bibliográfica se establecieron los valores de los parámetros del método EPIK (epikarst, cubierta protectora y paisaje kárstico) adaptándolos a las particularidades del estado de Yucatán. 
Para definir la infiltración, además de la revisión bibliográfica se realizaron pruebas de infiltración con el método del doble anillo, siguiendo el Protocolo de Infiltración (Globe, 1997). Se trazó un transecto que correspondió a los cinco paisajes geomorfológicos: planicie costera, planicie subhorizontal baja, dolinas agrupadas, planicie kárstica ondulada y planicie subhorizontal media. El criterio para ubicar el transecto fue incluir tanto los diferentes paisajes geomorfológicos del estado de Yucatán, como los distintos estados del desarrollo del karst.

Para llevar a cabo estas pruebas se siguió el Protocolo de Infiltración. La técnica descrita en el protocolo determina la tasa de infiltración midiendo el tiempo que toma que el nivel del agua que se deposita sobre el suelo penetre una cierta distancia (Globe, 1997). Las pruebas de campo se efectuaron de febrero a mayo de 2006, con un infiltrómetro de doble anillo. Los sitios para las pruebas de infiltración se eligieron considerando:a) zonas planas, donde el agua llega por escurrimiento superficial y finalmente se infiltra hacia el subsuelo, y b) la existencia de accesos adecuados que permitan realizar la prueba con las condiciones de seguridad requeridas. Posteriormente se analizaron las gráficas de infiltración por paisaje, y en cada paisaje se señaló la tendencia general de la infiltración y se hizo un análisis de varianza de una vía (Statgraphics Plus 5.I) para probar si hubo diferencias significativas entre los resultados de las pruebas.

\section{El método EPIK}

Los parámetros del método EPIK son: epikarst (características del epikarst); protection cover o cubierta protectora; infiltration conditions o infiltración;y karst network development o paisaje kárstico.

Una vez definidos los valores de los parámetros EPIK con base en las particularidades del estado de Yucatán, se evaluó la vulnerabilidad de acuerdo con el análisis multicriterio establecido por Doerfliger y Zwahlen (1998: 28). Según este método, a los parámetros se les asignan valores dependiendo de los rasgos que presenten. Existen cuatro factores de ponderación $(\alpha, \beta, \gamma, \delta)$ aplicables a cada parámetro antes mencionado. De aquí se obtiene el índice de vulnerabilidad intrínseca, también conocido como factor de protección:

$$
\mathrm{F}=\left(\alpha * \mathrm{E}_{i}\right)+\left(\beta * \mathrm{P}_{i}\right)+\left(\gamma * \mathrm{I}_{\mathrm{i}}\right)+\left(\delta * \mathrm{~K}_{i}\right)
$$

Donde:

$\mathrm{F}=$ factor de protección. 


$$
\begin{aligned}
& \mathrm{Ei}, \mathrm{Pi}, \mathrm{li}, \mathrm{Ki}=\text { valores relativos de los parámetros EPIK. } \\
& \alpha, \beta, \gamma, \delta=\text { factores de ponderación. }
\end{aligned}
$$

El cuadro I indica los valores relativos que se asignaron a los parámetros EPIK (Doerfliger y Zwahlen, 1998: 28).

Cuadro I.Valores relativos de los parámetros epik

\begin{tabular}{|l|l|l|l|l|l|l|l|l|l|l|l|l|l|}
\hline $\mathrm{E}_{\mathrm{I}}$ & $\mathrm{E}_{2}$ & $\mathrm{E}_{3}$ & $\mathrm{P}_{1}$ & $\mathrm{P}_{2}$ & $\mathrm{P}_{3}$ & $\mathrm{P}_{4}$ & $\mathrm{I}_{1}$ & $\mathrm{I}_{2}$ & $\mathrm{I}_{3}$ & $\mathrm{I}_{4}$ & $\mathrm{~K}_{\mathrm{I}}$ & $\mathrm{K}_{2}$ & $\mathrm{~K}_{3}$ \\
\hline $\mathrm{I}$ & 2 & 3 & $\mathrm{I}$ & 2 & 3 & 4 & $\mathrm{I}$ & 2 & 3 & 4 & $\mathrm{I}$ & 2 & 3 \\
\hline
\end{tabular}

$\mathrm{E}=$ Epikarst; $\mathrm{P}=$ Cubierta protectora; $\mathrm{I}=$ Infiltración; $\mathrm{K}=$ Paisaje kárstico.

Nota: Los subíndices expresan la clasificación de la vulnerabilidad para los diferentes parámetros. A valores menores la vulnerabilidad es mayor.

Fuente: Modificado de Doerfliger y Zwahlen (1998).

La vulnerabilidad aumenta conforme disminuyen los valores relativos; es decir, a valores relativos menores se considera que la vulnerabilidad es mayor. Doerfliger y Zwahlen (1998:28) propusieron que los factores de ponderación adopten los siguientes valores: $\alpha=3, \beta=1, \gamma=3, \delta=2$. El factor de protección puede variar entre 9 (más vulnerable) y 34 (menos vulnerable). La evaluación de la vulnerabilidad, de acuerdo con el valor del factor de protección $F$, queda de la siguiente manera: muy alta para una $F$ menor o igual a 19; alta para una $F$ entre 20 y 25; moderada para una $F$ mayor que 25; y baja para presencia de P4 (Doerfliger y Zwahlen, 1998: 31).

Para analizar la influencia de los parámetros EPIK en el valor del factor de protección $(F)$, se realizó una gráfica radial. También se efectuó un análisis de varianza simple (Statgraphics Plus 5.I) para probar si hubo diferencias significativas entre los valores obtenidos de la evaluación de la vulnerabilidad. Asimismo se compararon las medias de infiltración mediante un análisis de varianza (Anova) y la prueba de contraste múltiple de rango para probar la existencia de diferencias significativas mínimas, con la finalidad de identificar cuáles fueron las medias responsables de estas diferencias y así adecuar las categorías de vulnerabilidad obtenidas mediante el análisis multicriterio EPIK. 


\section{Resultados y discusión}

Epikarst

Se consideró como epikarst a la zona no saturada, ya que entre sus características principales se menciona que es una zona de infiltración rápida, con una profundidad, al nivel freático, que puede variar entre los 0.5 y los 30 metros.Además, es una zona que presenta karstificación excesiva, con fracturas y fisuras por las que el agua se infiltra rápidamente, y donde la capa más superficial es de roca caliza bien consolidada (denominada localmente "laja"), que tiende de manera importante al resquebrajamiento y la fragmentación. Por debajo de la laja se encuentra una capa de material calcáreo no consolidado (sascab), permeable, con alta capacidad de retención de humedad y con un espesor que va de uno a más de cinco metros.

Para emplear este parámetro en la evaluación de la vulnerabilidad en Yucatán, un factor adicional que se tuvo en cuenta fue la profundidad a la que se localiza el agua subterránea. Con base en este factor, la categoría $E_{1}$ puede considerarse dentro de los siguientes casos, de la más vulnerable a la menos vulnerable: $E_{11}$ cuando el agua subterránea se encuentra a una profundidad de entre uno y diez metros; $E_{12}$ cuando se halla entre $I 5$ y 30 metros; $y E_{13}$ cuando se ubica a más de 30 metros. En el cuadro 2 se presenta la clasificación del epikarst según el paisaje geomorfológico.

Cuadro 2. Clasificación para el parámetro E (Epikarst) del método EPIK, según el paisaje

\begin{tabular}{lcc}
\hline Paisaje geomorfológico ${ }^{1,2}$ & $\begin{array}{c}\text { Profundidad Nivel Estático } \\
(\mathrm{m})^{3}\end{array}$ & Clasificación para E \\
\hline Planicie costera & $1-5$ & $E_{11}$ \\
\hline Planicie kárstica subhorizontal baja & $5-10$ & $E_{11}$ \\
\hline Dolinas agrupadas abiertas & $1-10$ & $E_{11}$ \\
\hline Dolinas agrupadas cerradas & $5-20$ & $E_{11}$ \\
\hline Planicie kárstica ondulada & $15-30$ & $E_{12}$ \\
\hline Planicie kárstica subhorizontal media & $15-30$ & $E_{12}$
\end{tabular}

Fuente: Adaptado de: 'Bautista et al. (2005), ${ }^{2}$ Bautista et al. (2003), ${ }^{3}$ INEGI (2002), ${ }^{4}$ Doerfliger y Zwahlen (1998). 


\section{Cubierta protectora}

La cubierta protectora $(\mathrm{P})$ está formada por el suelo y otros elementos de cobertura. El método EPIK emplea el grosor de la cubierta protectora, es decir, el grosor del suelo, para evaluar la vulnerabilidad del acuífero (Doerfliger y Zwahlen, 1998: 21).

Para evaluar este parámetro se consideró el primer caso descrito por Doerfliger y Zwahlen (1998: 22), donde el suelo se sobrepone directamente sobre formaciones calcáreas, muy permeables; y se clasificó como:a) Categoría I (PI), que significa una cobertura de $0-20 \mathrm{~cm}$ de suelo; b) Categoría 2 (P2), que implica una cobertura de $20-100 \mathrm{~cm}$ de suelo; y c) Categoría 3 (P3), con una cobertura de más de $100 \mathrm{~cm}$ de suelo. En el cuadro 3 puede observarse la clasificación de la cubierta protectora según el paisaje geomorfológico.

\section{Cuadro 3. Clasificación para el parámetro P (Cubierta protectora)}

DEL MÉTODO EPIK, SEGÚN EL PAISAJE GEOMORFOLÓGICO

\begin{tabular}{|c|c|c|c|}
\hline Paisaje geomorfológico ${ }^{1,2}$ & Zonas edáficas $1,2,3$ & $\begin{array}{l}\text { Profundidad del suelo } \\
(\mathrm{cm})^{1,3}\end{array}$ & $\begin{array}{c}\text { Clasificación } \\
\text { para } \mathrm{P}^{4} \\
\end{array}$ \\
\hline Planicie costera & Regosol y Solonchak & $\begin{array}{l}>100 \\
<50\end{array}$ & $\mathrm{P}_{2}$ \\
\hline $\begin{array}{l}\text { Planicie kárstica subhorizontal } \\
\text { baja }\end{array}$ & Litosol y Rendzina & $\begin{array}{l}<10 \\
<30\end{array}$ & $P_{1}$ \\
\hline Dolinas agrupadas abiertas & Litosol y Rendzina & $<30$ & $P_{1}$ \\
\hline Dolinas agrupadas cerradas & Rendzina & $50-100$ & $P_{2}$ \\
\hline Planicie kárstica ondulada & Cambisol y Luvisol someros & $30-60$ & $P_{2}$ \\
\hline $\begin{array}{l}\text { Planicie kárstica subhorizontal } \\
\text { media }\end{array}$ & Cambisol y Luvisol profundos & $25-100$ & $\mathrm{P}_{2}$ \\
\hline
\end{tabular}

Fuente: Adaptado de 'Bautista et al. (2005), ${ }^{2}$ Bautista et al. (2003), ${ }^{3}$ Duch (1988), ${ }^{4}$ Doerfliger y Zwahlen (1998).

\section{Infiltración}

En el estado de Yucatán no se tienen valores documentados de infiltración, motivo por el cual se realizaron pruebas de campo siguiendo el Protocolo de Infiltración (GLOBE, 1997). En razón de que durante muchas de las pruebas efectuadas no se observó la estabilización de la tasa de infiltración, para evaluar este parámetro se utilizaron los valores promedio de cada sitio de prueba, en lugar del valor constante de la capacidad de infiltración (Fp) propuesto por 
Davies y de Wiest (197I: 66). Los valores promedio de la tasa de infiltración para cada prueba se obtuvieron sin tener en cuenta la medida inicial de la tasa de infiltración o tasa inicial de flujo (que es alta). El flujo se denomina no saturado mientras los poros de tierra seca no se han llenado de agua y no se ha alcanzado una tasa fija o estable, cuando ésta se presenta se considera flujo saturado.

En la planicie costera se observaron pruebas rápidas (entre cinco y 20 minutos), con dificultad para apreciar la estabilización del flujo, esto es, el flujo saturado. La tasa de infiltración promedio de la zona costera fue de 23.79 $\mathrm{mm} / \mathrm{min}$.

En la planicie kárstica subhorizontal baja se advirtió que a medida que transcurría el tiempo la tasa de flujo no saturado iba estabilizándose, aunque la estabilización (flujo saturado) no se observó por completo. El tiempo de las pruebas en este paisaje varió de seis a 45 minutos. De forma general, las pruebas llevadas a cabo en este paisaje mostraron una tendencia similar, que permitió apreciar la estabilización del flujo. La tasa de infiltración promedio de la planicie kárstica subhorizontal baja fue de $14.10 \mathrm{~mm} / \mathrm{min}$.

En las dolinas agrupadas abiertas no se hicieron pruebas de infiltración debido a que se asumió como el escenario más vulnerable, porque éstas se encuentran en contacto con el agua subterránea.

Por su parte, en las dolinas agrupadas cerradas se observó que el flujo comenzó a estabilizarse entre los 30 y los $45 \mathrm{~mm} / \mathrm{min}$, con tiempos de prueba que variaron entre dos y 60 minutos, lo que demostró la heterogeneidad de las características del suelo en un mismo sitio de prueba. Este paisaje presentó, en términos generales, una rápida tendencia a la estabilización de la tasa de infiltración, que en promedio fue de $43.50 \mathrm{~mm} / \mathrm{min}$.

En la planicie kárstica ondulada se encontró un caso de muy baja tasa de infiltración ( $3.37 \mathrm{~mm} / \mathrm{min})$; sin embargo, la tasa de infiltración promedio fue $18.09 \mathrm{~mm} / \mathrm{min}$. En este paisaje se observaron dos tendencias en las pruebas de infiltración:a) pruebas que alcanzaron los 45 minutos de duración, con tasas de infiltración promedio por prueba entre 2 y $6 \mathrm{~mm} / \mathrm{min}$ (la tasa promedio por sitios entre 3 y $5.6 \mathrm{~mm} / \mathrm{min}$ ), que pueden referirse como infiltración lenta, es decir, que el agua tardó en abrirse paso hacia el interior del suelo (a causa, entre otros, de la compactación del suelo); y b) pruebas de menor duración (entre uno y 15 minutos), con tasas de infiltración promedio entre 16 y $70 \mathrm{~mm} / \mathrm{min}$ 
(la tasa promedio por sitios entre 19 y $54 \mathrm{~mm} / \mathrm{min}$ ) que pueden designarse como infiltración más rápida.

En la planicie kárstica subhorizontal media, las pruebas de infiltración presentaron las mayores tasas de infiltración.Asimismo, se observaron pruebas de corta duración, (entre dos y 5:30 minutos), con altas tasa de flujo, y pruebas de mayor duración (entre II y 45 minutos) con tasas menores. En ambos casos se pudo apreciar la estabilización de la tasa de infiltración.A este paisaje correspondieron la mayor tasa de infiltración promedio registrada (I76.75 $\mathrm{mm} / \mathrm{min}$ ) y el mayor valor promedio por sitio $(130.4 \mathrm{I} \mathrm{mm} / \mathrm{min})$. La tasa de infiltración promedio de este paisaje fue $65.18 \mathrm{~mm} / \mathrm{min}$.

Se advirtió que las mayores tasas promedio de infiltración se obtuvieron en zonas donde el suelo es más profundo.

Para clasificar este parámetro (cuadro 4) se tomaron en consideración la tasa de infiltración y la estabilización del flujo. Las pruebas de corta duración con altas tasas de infiltración y en las que se no se observó la estabilización del flujo se clasificaron como $\mathrm{I}_{1}$; mientras que las pruebas de mayor duración con tasas de infiltración menores y en las que se apreció la estabilización del flujo se clasificaron como $\mathrm{I}_{2}$; $\sin$ embargo, las veces en que no se observó la estabilización del flujo durante las pruebas, las tasas de infiltración se clasificaron como I.Asimismo, se aplicó un análisis de varianza (Anova), para identificar por medio de los valores de infiltración los paisajes con diferencias significativas, y resultaron dos grupos homogéneos: el primero formado por las planicies costera, kárstica subhorizontal baja y kárstica ondulada; y el segundo, por las dolinas agrupadas y la planicie kárstica subhorizontal media.

Cuadro 4. Clasificación de la inflLtración (I) Y el paisaje kárstico (K) del MÉTODO EPIK, POR PAISAJE GEOMORFOLÓGICO

\begin{tabular}{lccc}
\hline Paisaje geomorfológicol,2 & $\begin{array}{c}\text { Infiltración } \\
(\mathrm{mm} / \mathrm{min})\end{array}$ & $\begin{array}{c}\text { Clasificación } \\
\text { para I }\end{array}$ & $\begin{array}{c}\text { Clasificación } \\
\text { para K}^{3}\end{array}$ \\
\hline Planicie costera & 23.79 & $\mathrm{I}_{1}$ & $\mathrm{~K}_{4}$ \\
Planicie kárstica subhorizontal baja & $\mathrm{I} .10$ & $\mathrm{I}$ & $\mathrm{K}_{3}$ \\
Dolinas agrupadas abiertas & $\mathrm{nm}$ & $\mathrm{I}_{1}$ & $\mathrm{~K}_{1}$ \\
Dolinas agrupadas cerradas & 43.50 & $\mathrm{I}_{2}$ & $\mathrm{~K}_{1}$ \\
Planicie kárstica ondulada & 18.09 & $\mathrm{I}_{2}$ & $\mathrm{~K}_{2}$ \\
Planicie kárstica subhorizontal media & 65.18 & $\mathrm{I}_{1}$ & $\mathrm{~K}_{3}$
\end{tabular}

Fuente: Adaptado de 'Bautista et al. (2005), ${ }^{2}$ Bautista et al. (2003), ${ }^{3}$ Doerfliger y Zwahlen (1998). Nota: $\mathrm{nm}=$ no medido. 


\section{Paisaje kárstico}

El desarrollo del karst (K) es el conjunto de expresiones kársticas, como cenotes y cavernas, incluyendo las fracturas, fisuras, conductos y canales preferenciales de flujo, formados como resultado de la disolución de las rocas calizas.

El método EPIK evalúa la vulnerabilidad en términos de la presencia o ausencia del paisaje kárstico $(K)$ y del grado de desarrollo que éste presenta. Para identificar este parámetro se emplearon diversos indicadores; uno de ellos, el más adecuado conforme a las características de las planicies del estado de Yucatán, es la identificación directa de los componentes del paisaje kárstico: cuevas y agujeros de infiltración. Las expresiones más representativas del paisaje kárstico yucateco son: cavernas, cenotes, rejolladas y aguadas.

Como muestra del desarrollo del paisaje kárstico yucateco, Springall y Espinosa (1972: 40) encontraron al norte de la ciudad de Mérida cavidades de distintas magnitudes y profundidades. Durante sus exploraciones en diferentes lugares de la ciudad, observaron un elevado número de cavidades y conductos de disolución producto del fenómeno kárstico. Asimismo, afirmaron que la probabilidad de encontrar cavidades en los primeros cinco metros es de 52 $\%$. En perfiles del subsuelo en la zona henequenera, advirtieron cavidades a diversas profundidades. (Springall y Espinosa, 1972:44). Con respecto a la zona costera, los autores comentaron que los efectos del fenómeno kárstico pueden apreciarse a simple vista, como los manantiales que emergen en las costas $y$ en el fondo del mar por medio de los conductos de disolución.

Una prueba más del buen desarrollo del paisaje kárstico yucateco está expresada en el trabajo de Perry et al. (1995: 18), quienes encontraron en el noroeste de la Península una buena comunicación del acuífero y las rocas altamente permeables, lo que se hace patente en la rápida recuperación del nivel del agua subterránea después de la lluvia; así como la influencia del anillo de cenotes en el flujo local. Los autores afirman que el anillo de cenotes actúa como un canal de flujo, de tal forma que el agua que fluye hacia el anillo de cenotes por el noroeste se mueve hacia el estuario de Celestún y por el noreste hacia las Bocas de Dzilam. Esto lo aseguran tanto con base en evidencia química, como en la presencia de abundantes arroyos submarinos (ojos de agua) en las costas del estuario de Celestún y en las Bocas de Dzilam. 
Otras manifestaciones del buen desarrollo del paisaje kárstico son la alta transmisividad del acuífero (con un bajo gradiente hidráulico, $0.007 \mathrm{~m} / \mathrm{km}$ ) debido al sistema kárstico, a las fracturas existentes en la región y a que las pruebas de bombeo no tienen el éxito esperado, dada la rápida estabilización del nivel dinámico del agua mientras se realiza la prueba (Sánchez-Pinto, González-Herrera y Perry, 2005: 9).

Para aplicar el método EPIK al acuífero en el estado de Yucatán, se asignaron las siguientes categorías a los paisajes geomorfológicos:

- Categoría I (KI), dolinas agrupadas: este elemento del paisaje kárstico representó la mayor vulnerabilidad porque se encuentra en contacto con el agua subterránea, además de que en este paisaje se asume un buen desarrollo kárstico por las fracturas y los conductos de flujo preferenciales que caracterizan a estos elementos kársticos.

- Categoría 2 (K2), planicie kárstica ondulada: aquí se halla una gran variedad de elementos del paisaje kárstico y abundantes fracturas.

- Categoría 3 (K3), planicies kársticas subhorizontal baja y media: paisajes con características y estado de desarrollo del karst similares.

- Categoría 4 (K4), planicie costera:presenta una capa impermeable de caliza recristalizada que confina las aguas subterráneas a lo largo de la costa de Yucatán (Bautista et al., 2005:45; Sánchez-Pinto, González-Herrera y Perry, 2005: II). (Cuadro 4.)

\section{Evaluación de la vulnerabilidad}

Una vez establecidas las características del estado de Yucatán conforme a los parámetros del método EPIK, se les asignaron los valores del cuadro 5.

\section{Cuadro 5.VAlores a parámetros epIK}

\begin{tabular}{|l|l|l|l|l|l|l|l|l|l|}
\hline $\mathrm{E}_{11}$ & $\mathrm{E}_{12}$ & $\mathrm{P}_{1}$ & $\mathrm{P}_{2}$ & $\mathrm{I}$ & $\mathrm{I}_{2}$ & $\mathrm{~K}_{1}$ & $\mathrm{~K}_{2}$ & $\mathrm{~K}_{3}$ & $\mathrm{~K}_{4}$ \\
\hline $\mathrm{I}$ & 2 & $\mathrm{I}$ & 2 & $\mathrm{I}$ & 2 & $\mathrm{I}$ & 2 & 3 & 4 \\
\hline
\end{tabular}

$\mathrm{E}=$ Epikarst; $\mathrm{P}=$ Cubierta protectora; $\mathrm{I}=$ Infiltración; $\mathrm{K}=$ Paisaje kárstico.

Nota: Los subíndices expresan la clasificación de la vulnerabilidad para los diferentes parámetros. A valores menores la vulnerabilidad es mayor. 
Los valores de los factores de ponderación empleados fueron los propuestos por Doerfliger y Zwahlen (1998: 28). A continuación se presenta la evaluación de la vulnerabilidad del agua subterránea, mediante el método EPIK. Aunque la mayoría de los sitios evaluados se consideran muy vulnerables, se puede observar que esta vulnerabilidad se manifiesta en diversos grados en los diferentes paisajes. El paisaje más vulnerable es el que obtuvo el menor valor promedio del factor de protección (F), que correspondió a dolinas agrupadas con un valor $\mathrm{F}$ promedio de 13 ; $y$ el menos vulnerable fue la planicie kárstica subhorizontal media con un valor de F promedio de 18.29. La influencia de los distintos parámetros es diferente para cada paisaje. La figura 2 exhibe los valores relativos de la vulnerabilidad con respecto a los factores de ponderación EPIK por cada paisaje. La escala empleada es de 0 a $100 \%$, donde los valores que representan la mayor vulnerabilidad se señalan con $100 \%$, y con 75,50 o $25 \%$ los valores proporcionales conforme la vulnerabilidad disminuye.

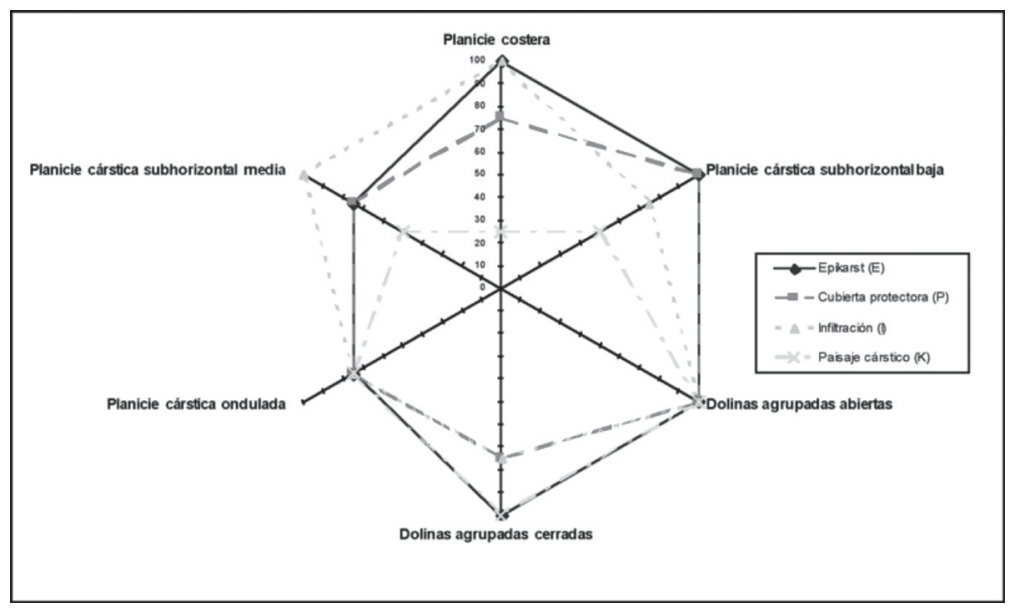

Figura 2. Gráfica Radial de valores epiK Relativos para cada paisaje

La variación del valor del factor de protección (F) se debe a una combinación de factores que dependen de las características del paisaje. De acuerdo con los valores relativos, el comportamiento de los parámetros EPIK puede referirse como de muy alta importancia si el valor relativo representa la mayor vulnerabilidad, y como de importancia alta, media y baja conforme la vulnerabilidad disminuye. En este sentido tenemos: 
a) En las dolinas agrupadas abiertas todos los parámetros fueron de muy alta importancia, de ahí se obtuvo el paisaje con la mayor vulnerabilidad.

b) El epikarst fue un factor de muy alta importancia en tres de los cinco paisajes muestreados (planicie costera, planicie kárstica subhorizontal baja y dolinas agrupadas).

c) Por su parte, la cubierta protectora tuvo muy alta importancia en la planicie kárstica subhorizontal baja y las dolinas agrupadas abiertas; $y$ en los paisajes restantes (planicie costera, planicie kárstica ondulada, dolinas agrupadas cerradas y planicie kárstica subhorizontal media) fue un factor de alta importancia.

d) La infiltración mostró ser un factor de muy alta importancia en dos de los cinco paisajes muestreados: planicie costera y planicie kárstica subhorizontal media (en este último fue el factor que indicó la mayor vulnerabilidad); mientras que en los tres paisajes restantes (planicie kárstica ondulada, planicie kárstica subhorizontal baja y dolinas agrupadas), la infiltración fue un factor de alta importancia.

e) El paisaje kárstico tuvo muy alta importancia para las dolinas agrupadas, alta importancia para la planicie kárstica ondulada, importancia media para las planicies kársticas subhorizontales baja y media, y baja importancia para la planicie costera.

De acuerdo con el análisis multicriterio del método EPIK, se obtuvo el factor de protección $(\mathrm{F})$ para cada sitio de pruebas de infiltración y características del paisaje geomorfológico. En el cuadro 6 se aprecian los resultados de la evaluación de la vulnerabilidad en las planicies de Yucatán.

Según el factor de protección obtenido, la vulnerabilidad para los sitios muestreados es muy alta, a excepción de tres sitios de la planicie kárstica subhorizontal media donde fue evaluada como alta.

Mediante el método de la diferencia significativa mínima se compararon las medias para descubrir cuáles son las causantes de las diferencias significativas, ya que se identificaron tres grupos homogéneos.

A partir de los resultados, se propone una adecuación de las categorías obtenidas del análisis multicriterio EPIK (cuadro 6 y figura 3). Esta nueva clasificación tuvo como base los valores del factor de protección $(F)$ y el resultado del contraste múltiple de rango aplicado a estos valores, que probó 
la existencia de diferencias significativas entre los grupos formados por las dolinas agrupadas; las planicies kárstica subhorizontal baja, costera y kárstica ondulada; y la planicie kárstica subhorizontal media.

Cuadro 6. Evaluación de la VULNerabilidad en las planicies del estado de YuCatán

\begin{tabular}{|c|c|c|c|c|c|c|c|}
\hline \multirow[b]{2}{*}{ Paisaje $^{1,2}$} & \multicolumn{4}{|c|}{ Método 3} & \multirow{2}{*}{$\begin{array}{l}\text { Factor de } \\
\text { Protección } \\
(\mathrm{F})^{3}\end{array}$} & \multicolumn{2}{|c|}{ Vulnerabilidad } \\
\hline & $\mathrm{E}$ & $P$ & 1 & K & & Método EPIK ${ }^{3}$ & $\begin{array}{l}\text { Adaptación } \\
\text { a Yucatán }\end{array}$ \\
\hline P. Costera & I & 2 & I & 4 & 16.0 & Muy Alta & Alta \\
\hline P. kárstica subhorizontal baja & 1 & 1 & 2 & 3 & 1.06 & Muy Alta & Alta \\
\hline Dolinas agrupadas cerradas & $\mathrm{I}$ & 2 & 2 & 1 & 13.0 & Muy Alta & Muy Alta \\
\hline Dolinas agrupadas abiertas $(\mathrm{nm})$ & 1 & I & I & 1 & 9.0 & Muy Alta & Extrema \\
\hline P. kárstica ondulada & 2 & 2 & 2 & 2 & 16.5 & Muy Alta & Alta \\
\hline P. kárstica subhorizontal media & 2 & 2 & I & 3 & 18.29 & Muy Alta & Media \\
\hline
\end{tabular}

$\mathrm{E}=$ Epikarst; $\mathrm{P}=$ Cubierta protectora; $\mathrm{I}=$ Infiltración; $\mathrm{K}=$ Paisaje kárstico.

Nota: $\mathrm{nm}=$ no muestreado.

Fuente: Adaptado de 'Bautista et al. (2005), ${ }^{2}$ Bautista et al. (2003), ${ }^{3}$ Doerfliger y Zwahlen (1998).

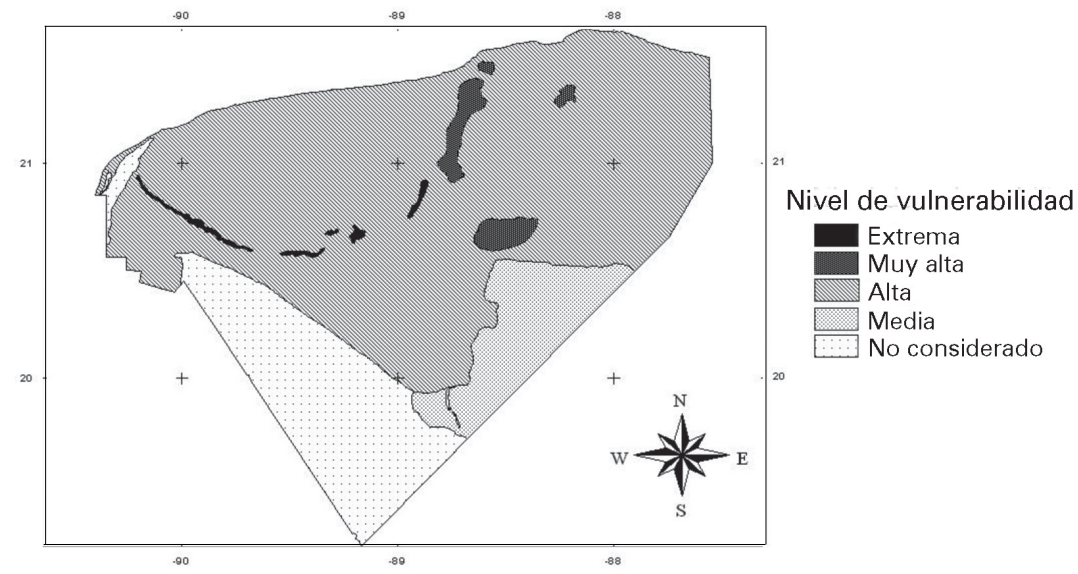

Figura 3. Mapa de vulnerabilidad de contaminación del agua subterRánea en YuCatán, MéXICO. 


\section{Conclusiones}

Por sus características, la zona no saturada del estado de Yucatán puede considerarse como epikarst, por lo que se asoció con la categoría $\mathrm{E}_{\text {, del }}$ método EPIK.

Las características kársticas y el tipo de suelo tuvieron una influencia importante en los valores de la infiltración, y confirmaron la heterogeneidad de estas características en ambientes kársticos.

El estado de Yucatán muestra un buen desarrollo del paisaje kárstico representado por dolinas agrupadas, planicies kársticas onduladas y planicies kársticas subhorizontales, donde la influencia del paisaje kárstico para determinar el factor de protección (F) fue muy alta, alta y media, respectivamente.

La evaluación realizada al aplicar el método EPIK evidenció que en el estado de Yucatán el acuífero es muy vulnerable a la contaminación. Al paisaje geomorfológico de dolinas agrupadas le correspondió la categoría más vulnerable.

La mayoría de los paisajes se clasificó como muy vulnerable; sin embargo, con el desglose del índice se pudo observar que los valores de los parámetros EPIK no son idénticos, y que es la combinación de éstos la que produce resultados semejantes.

La aplicación del método EPIK permitió evaluar la vulnerabilidad del agua subterránea en esta área mediante la adaptación de los parámetros involucrados conforme a las características kársticas del estado de Yucatán, por lo cual se llevó a cabo una evaluación más adecuada.

\section{Recomendaciones}

Aplicar el método en áreas en donde se cuente con información más detallada acerca de los parámetros EPIK, especialmente de la infiltración (I) y la cubierta protectora $(\mathrm{P})$, para proporcionar recomendaciones más acertadas respecto a la protección del agua subterránea y el manejo de suelo para las diferentes actividades humanas. 
Asimismo, realizar un mayor número de pruebas de infiltración en los distintos paisajes geomorfológicos, para clasificar con mayor precisión este parámetro.

Debido a que la diversidad de suelos en el estado tuvo una importante influencia en las variaciones del valor del factor de protección $\mathrm{F}$, se recomienda revisar de manera detallada la clasificación de la cubierta protectora para que refleje las características de los suelos de la región.

\section{Agradecimientos}

Al Fondo Mixto Consejo Nacional de Ciencia y Tecnología (Conacyt)-Gobierno de Yucatán, por el apoyo al proyecto Base de Datos Digitales del Medio Físico para la Creación de las Zonas Agroecológicas del Estado de Yucatán" (Clave YUC-2003-CO2-054). Emilio Bolio agradece al Conacyt la beca otorgada para estudios de posgrado.

\section{FUENTES CONSULTADAS}

Auge, M. (2004). "Vulnerabilidad de acuíferos". Revista Latinoamericana de Hidrogeología, 4, 85- 103.

Bautista, F., et al. (2003). "Geoformas, agua y suelo en la Península de Yucatán”, en P. Colunga-García Marín y A. Larqué-Saavedra (eds.). Naturaleza y sociedad en el área maya: pasado, presente y futuro. México: Centro de Investigaciones Científicas de Yucatán/Academia Mexicana de Ciencias, 21 -34.

(2005). "Integración del conocimiento actual sobre los paisajes geomorfológicos de la Península de Yucatán”, en F. Bautista y G. Palacio (eds.). Caracterización y manejo de los suelos de la Península de Yucatán. Implicaciones agropecuarias, forestales y ambientales. México: Universidad Autónoma de Campeche/Universidad Autónoma de Yucatán (UADY)/Instituto Nacional de Ecología, 33-58.

Daly, D., et al. (2002). "Main Concepts of the 'European Approach' for (Karst) Groundwater Vulnerability Assessment and Mapping”. Hydrogeology Journal, I0, 340-345.

Davies, S. N. y R. de Wiest (I97I). Hidrogeología. Barcelona:Ariel, 563 p. Doerfliger, N. y F. Zwahlen (1998). Groundwater Vulnerability Mapping in Karstic Regions (EPIK). Practical Guide. Berna:Swiss Agency for the Environment, Forest and Landscape, 56 p. 
Doerfliger, N., P. Jeannin y F. Zwahlen (1999). “Water Vulnerability Assessment in Karst Environments: A New Method of Defining Protection Areas Using a Multi-attribute Approach and gis tools (EPIK method)". Environmental Geology, 39 (2), I65-176.

Duch, J. (1988). La conformación territorial del estado de Yucatán. México: Universidad Autónoma Chapingo/Centro Regional de la Península de Yucatán, $427 \mathrm{p}$.

Flores, S. e I. Espejel ( 1994). Etnoflora yucatanense. Fascículo 3: Tipos de vegetación de la Península de Yucatán. Mérida, Yucatán: uady.

GLOBE (1997). "Protocolo de Infiltración". Investigación de suelos. Una investigación de aprendizaje de GLOBE [en línea]. Disponible en: http:// www.globe.gov/sda/tg97es/suelos/Suelos.pdf [2006, enero].

Goldscheider, N. (2005).“"Karst GroundwaterVulnerability Mapping:Application of a New Method in the Swabian Alb, Germany”. Hydrological Journal, 13, 555-564.

Ihl,T., et al.(2007).“Identification of Geodisasters in the State of Yucatan, Mexico". Neues Jahrbuch für Geologie und Paläontologie, 246 (3), 299-3II.

INEGI (2002). Estudio Hidrológico del Estado de Yucatán. Aguascalientes: Instituto Nacional de Estadística, Geografía e Informática.

Orellana Lanza, R., et al. (1999).“Evaluación climática”, en A. García y J. Córdoba (eds.).Atlas de procesos territoriales deYucatán. Mérida,Yucatán: Facultad de Arquitectura-uady, I63-182.

Pérez, R.y J.Pacheco (2004).“Vulnerabilidad del agua subterránea a la contaminación de nitratos en el estado de Yucatán”. Ingeniería, 8 (I), 33-42.

Perry, E., et al. (1995). "Ring of Cenotes (Sinkholes), Northwestern Yucatan, Mexico: Its Hydrogeologic Characteristics and Possible Association with the Chicxulub Impact Crater". Geology, 23 (I), I 7-20.

Sánchez-Pinto, I., R. González-Herrera y E. Perry (2005). "Hydrodynamic Behavior of the Yucatan Aquifer. A Perspective on the Hydraulic Conductivity Estimation”. Espelunc@ Digital. Órgano Oficial de la Sociedad Espeleológica de Cuba, 2, 8-20.

Springall, G. y L. Espinosa (1972). "El subsuelo de la Península de Yucatán”. Memoria de la VI Reunión Nacional de Mecánica de Suelos. México. 